\title{
REVIEWS
}

EVOLUTION UND HOMINISATION. Edited by G. Kurth. Stuttgart: G. Fischer. 1962. Pp. 228, 47 figs. $48.50 \mathrm{DM}$.

Eighteen collected papers are here dedicated to Gerhard Heberer. They concern evolution, systematics and the fossil history of man. They are mostly specialised and disconnected and somewhat contradictory of one another.' Some also concern the problem of whether the genera Australopithicus, Pithecanthropus, Pavanthropus, Sinanthropus, Javanthropus, Africanthropus, Cyphanthropus, Atlanthropus, Telanthropus, Palaeanthropus and Euranthropus might not all prove to be just Homo if we knew the whole truth. Examining the dating of early human remains, Kurtén suggests that man in Java may be as old as in South Africa but he agrees that the type of early man in South Africa is the earlier. We are therefore left with the view that man probably began in Africa and afterwards moved into Asia.

Genetic problems are not put in the foreground of these discussions except in the paper of Robinson with which those of Dart and Oakley are connected. Robinson argues in favour of the view that man's inventions have directed the course of his evolution by changing his selective situation. His interaction with his culture can thus be expressed in purely biological terms. This principle seems to be fundamental for human biology since it applies equally whether the invention is the use of bone implements, of the cultivation of crops or of the smelting of metals : each of these changed the selective forces at work to the advantage of the men who made the invention.

\section{D. Darlington.}

BIOLOGICAL ALKYLATING AGENTS. By W. C. J. Ross. London: Butterworths. 1962. Pp. 232. E2, 15s.

Approximately half of this book deals with the fundamental chemistry of alkylating agents and their reactions with biologically occurring compounds. The remainder is concerned with the use of such reagents in the treatment of cancer, the problem being considered in general terms from the standpoint of selective toxicity. It is pleasing to see here the application of certain fundamental biochemical studies as exemplified by the modification of carcinostatic or carcinocidal substances to result in preferential uptake by cancer cells or hydrolysis to liberate the toxic compound in situ.

This is the first monograph dealing with these reagents and the book's greatest value will be for those concerned with the chemical development and testing of selectively toxic substances. For the geneticist the brief section discussing the effect of alkylation on nucleic acids will be most interesting.

K. W. Fuller.

INTRODUCTION TO THE MATHEMATICAL THEORY OF GENETIC LINKAGE. BY N. T. J. Bailey. Oxford: At the Clarendon Press. 1961. Pp. 298. 55s.

Since the primary purpose of this book is " to be of assistance to those geneticists who require to use mathematical methods", and since it goes by the name of an introduction to the subject of linkage, one might be 
forgiven for supposing that it will take the suspicious biologist by the hand and lead him gently through the mathematics of linkage estimation and genetic map-making. But before the journey is half over he will have stumbled on an integral "where $c$ is positive and greater than the abscissæ of all the singularities of the integrand ", a condition which, for its comprehension, requires at least a greater knowledge of mathematics than the reviewer possesses. So beware! The path is well-signposted, but the place names include Vector, Matrix, Jacobian, and Laplace Transform. This, however, is a criticism of the title rather than of the book. For Dr Bailey's book is no less than a thorough account of the theory of linkage : it leaves few stones unturned, and combines mathematical rigour with clarity of exposition to an unusual degree.

The book is divided into four parts. Part I is introductory, and provides an excellent account of basic genetics from the mathematical standpoint. Part II covers the detection and estimation of linkage in experimental populations, and, apart from the chapter on polysomic inheritance, needs no advanced mathematical knowledge: it should serve as a welcome handbook for the practising geneticist. Part III is devoted to a lucid account of the theory of chromosome mapping, but, being rather difficult mathematically, will probably-and unfortunately-only appeal to specialists in this field. In Part IV the author considers linkage in human populations : after a good account of ascertainment, in which field he is particularly at home, he considers Fisher's $u$-statistics, the problem of partial manifestation, and Smith's "odds" approach to pedigree analysis. Penrose's sib-pair and Morton's sequential methods are barely mentioned, so that the human geneticist will not derive such practical benefit from the book as his experimental colleague.

The reviewer, being, like Dr Bailey, a product of R. A. Fisher's Department at Cambridge, is too close to the subject-matter of the book to be able to make an unbiased comment on its importance to contemporary genetics. Indeed, some aspect of the work of Fisher and his students occurs on nearly every page, from the simplest linkage estimation formulæ, to linkage in polysomics, chromosome mapping, the segmental calculus, ascertainment, and the $u$-statistics. And pervading all is the method of maximum likelihood and Fisher's variance formula (each of which has an appendix to itself), as well as $\chi^{2}$. The book is thus a fitting epilogue to the linkage work of Fisher and his school at Cambridge, whose members are now dispersed to the four corners of the earth.

But even if a general appraisal is not possible, some detailed comments are. The care with which Dr Bailey has evidently examined original papers is most commendable: he expounds their arguments critically, corrects their errors, and, in many cases, has clearly redone the computations as well; nor is he afraid to admit that there are some obscure points he does not fully understand. He is at pains to justify biological mathematics in the eyes of pure mathematicians, and goes so far as to state that "the scope for mathematical skill and ability is at least as great as that available in the more exact sciences". Even the most enthusiastic biometrician will surely regard this as an excessive claim.

In a discussion of the causes of disturbed segregations (p. I3), it is a pity that abnormal meiotic ratios are not mentioned, for these are probably more widespread than formerly believed. On page $15^{2}$ we learn that 
Kosambi's addition formula for recombination fractions is similar to the velocity addition rule in the special theory of relativity, " though no particular significance seems to attach to the correspondence". This is perhaps an understatement, for the mathematical coincidence reflects a real similarity between the two problems. Do physicists realise that, if they measure velocity on the scale $\tanh ^{-1} 2 \mathrm{v}$, where $v$ is in units of the velocity of light, then it is additive under the special theory of relativity?

In view of the exactitude with which recombination fractions can be estimated with the aid of the theory of this book, it is surprising that Dr Bailey has not considered the overestimation of linkage that must sometimes arise through preceding the estimation procedure by a test of signifcance for linkage: one does not estimate from non-significant data. It might be a better practice to estimate the recombination fraction and then to test whether it differs significantly from one-half. Otherwise we will never have a linkage of 49 per cent. for the individual sets of data, which, when combined, produce this value, are all forgotten !

The quoting of recombination fractions in percent leads to values such as " $26 \cdot 03 \pm I \cdot 96$ per cent", which is ambiguous, and needs a clarifying statement somewhere. The only other point of presentation to which one might take exception is the author's frequent use of the abbreviation "i.e." as if it were a conjunction. As there are well over six hundred equations and formulæ in the book, the reviewer hopes he may be excused from checking them. The typography is in accordance with the fine tradition of Oxford mathematical printing, and only one misprint was noticed : an entirely trivial one on page 135, where the expression for $\mathrm{y}_{2}$ contains a full-stop instead of a point !

Here, then, is an elegant treatise on a biological subject for applied mathematicians, a wealth of examples on the power of the method of maximum likelihood for statisticians, a complete handbook on linkage for bio-mathematicians, and the only unified account of interference theory extant. But " those geneticists who require to use mathematical methods", if their training has been essentially biological, would still be well-advised to start with Professor Mather's monograph on the measurement of linkage, which does not assume such an extensive knowledge of mathematics and statistics on the part of the reader.

A. W. F. EDWARDs. 